\section{Paradoxical Nodular Scleritis during Tocilizumab Therapy: A Case Report}

\section{To the Editor:}

Ocular manifestations can result from interleukin 6 (IL-6) inhibition, and they should be considered as a possible paradoxical complication when treating inflammatory arthritis.

We report the case of a 48-year-old woman with a background history of rheumatoid arthritis (RA), pyoderma gangrenosum, and systemic lupus erythematosus who was diagnosed with anterior nodular scleritis following initiation of tocilizumab (TCZ) therapy. Her RA remained active despite being treated with rituximab (RTX) 2 g intravenously every 6 months, methotrexate (MTX) $20 \mathrm{mg}$ orally every week, sulfasalazine $1 \mathrm{~g}$ orally twice daily, hydroxychloroquine $200 \mathrm{mg}$ orally once daily, and folic acid $5 \mathrm{mg}$ orally every week. She was switched to TCZ $8 \mathrm{mg} / \mathrm{kg}$ intravenously every 4 weeks. RTX was stopped 5 months prior to TCZ because of primary failure. MTX was discontinued immediately prior to TCZ owing to government funding requirements whereby TCZ can only be given as monotherapy. Following the first dose of TCZ, her RA went into clinical remission, with a 28 -joint Disease Activity Score of 2.41 from 6.28 prior. In addition, the inflammatory markers were at a nadir (C-reactive protein went from 28 to $<1$ ) not seen in 2 years. Emergence of the lesion shown in Figure 1 occurred following the second dose of TCZ. A clinical diagnosis of nodular scleritis was formulated and this responded to oral prednisone: a dose of $20 \mathrm{mg}$ daily tapered over 3 months. Moreover, concomitant withdrawal of TCZ therapy saw complete resolution of the nodular lesion at 9 months (Figure 2), while she took no steroids. Ophthalmological examination using the slit-lamp showed no other signs of intraocular inflammation. A full blood count, tuberculosis blood test, and Treponema screen were performed, and infectious etiology excluded. It was posited that this was a paradoxical effect of the TCZ treatment, given that the patient's inflammatory profile was suppressed, she had never experienced extraarticular manifestations before, and considering the temporal relationship with commencement of TCZ.

Paradoxical reactions may be defined as "the occurrence with therapy with a biological agent, of a pathological condition that usually responds to this class of drug ${ }^{1}$." This may be further subclassified into true reactions: for example, the onset of de novo psoriasis during RTX therapy (or in parallel - the exacerbation of a preexisting condition upon starting a biological agent), or borderline reactions. The latter exist when a biological agent has a theoretical rationale for its efficacy in treating a certain condition (but is not registered for it), yet gives rise to the development of that condition ${ }^{1}$.

TCZ is an anti-IL-6 receptor monoclonal antibody, thereby conferring

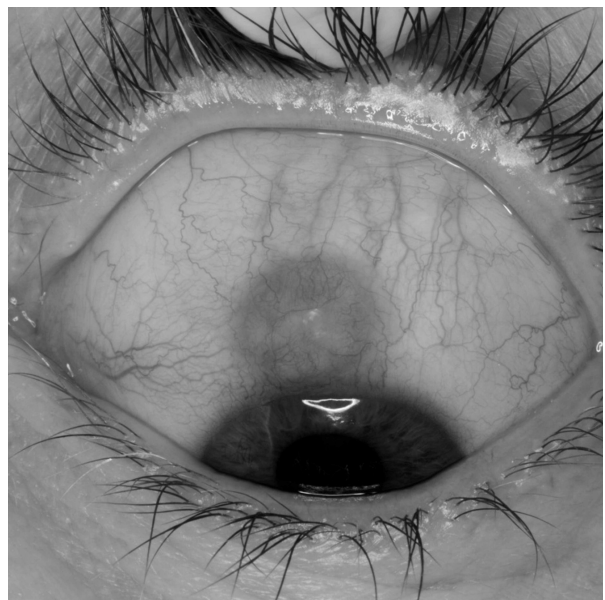

Figure 1. A 4-mm raised scleral nodule in the superlimbal region was noted following tocilizumab therapy. its antiinflammatory properties through this mechanism. Elevated levels of IL-6 have been detected in the eyes of patients with refractory uveitis and thus TCZ may have an important role in the treatment of ocular inflammation ${ }^{2}$. TCZ has demonstrated efficacy in the treatment of RA ${ }^{3}$, recalcitrant uveitis and its associated macular edema ${ }^{2}$, and treatment-refractory scleritis ${ }^{4,5,6}$. It is approved for usage by the US Food and Drug Administration in RA and in polyarticular or systemic juvenile idiopathic arthritis $^{2}$

Case reports of paradoxical reactions with TCZ therapy have been documented. Laurent, et al mentions 2 instances of psoriatic reactivation following TCZ initiation ${ }^{7}$. Similarly, a single account was noted of a patient developing psoriasiform palmoplantar pustulosis when having TCZ for treatment of RA. The rash abated upon discontinuation of therapy ${ }^{8}$. A possible case report of TCZ-induced sarcoidosis has also been described in a 40-year-old woman with RA, in whom other biological agents (etanercept and certolizumab) had been used without such incidence .

Despite these reports, the onset of ocular inflammation with TCZ is rarely reported in literature. In 1 anecdote, a 41-year-old man with HLA-B27-positive ankylosing spondylitis experienced his first-ever episode of acute anterior uveitis between the second and third doses of TCZ. In

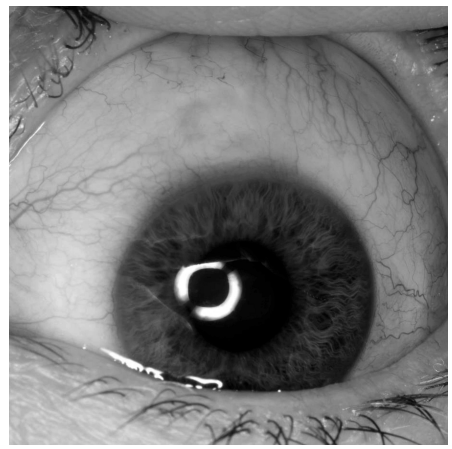

Figure 2. Complete resolution of scleral nodule at 9 months following the cessation of tocilizumab therapy.

another report, a 65-year-old man developed peripheral ulcerative keratitis following 11 months of TCZ. Systems review and infection screening were unyielding. Relapse of keratitis occurred 20 days after the third infusion, therefore TCZ was withheld for 2 months. After reintroduction of therapy 19 days later, a third episode of keratitis occurred. In these 2 cases, it is worth considering that inflammatory eye disease is not paradoxical but an extraarticular feature of their rheumatological conditions. However, these manifestations had not occurred prior to TCZ, but during a period in which systemic inflammation was well controlled. In addition, a favorable systemic clinical response to TCZ was observed. Other causes of inflammation (such as infection) were excluded and in the case of keratitis, the rechallenge test was positive ${ }^{10}$.

Biological agents such as TNF antagonists may precipitate de novo uveitis or scleritis in situations where the patient's rheumatological condition is controlled by such treatment. To our knowledge, this is the first example of nodular scleritis following TCZ therapy. A rechallenge of TCZ was offered to our patient, but she declined. We conclude that ocular manifestations as a result of IL-6 inhibition should be considered as a possible paradoxical phenomenon when treating inflammatory arthritis.

EUGENE MICHAEL, MBChB, BPharm, Ophthalmology Registrar, Meade Clinical Centre, Department of Ophthalmology, Waikato District Health Board; EVA L. LANGE, MBChB, FRACP, Meade Clinical Centre, Department of Rheumatology, Waikato District Health Board; STEPHEN GUEST, MBBS, FRANZCO, Meade Clinical Centre, Department of Ophthalmology, Waikato District Health Board, Hamilton, New Zealand. Address correspondence to Dr. E. Michael, Greenlane Clinical Centre, Department of Ophthalmology, Auckland District Health Board, Auckland, New Zealand. E-mail: EugeneM@adhb.govt.nz 


\section{REFERENCES}

1. Toussirot E, Aubin F. Paradoxical reactions under TNF- $\alpha$ blocking agents and other biological agents given for chronic immune-mediated diseases: an analytical and comprehensive overview. RMD Open 2016;2:e000239.

2. Mesquida M, Leszczynyska A, Llorenc V, Adan A. Interleukin-6 blockade in ocular inflammatory disease. Clin Exp Immunol 2014;176:301-9.

3. Yamanaka H, Tanaka Y, Inoue E, Hoshi D, Momohara S, Hanami K, et al. Efficacy and tolerability of tocilizumab in rheumatoid arthritis patients seen in daily clinical practice in Japan: results from a retrospective study (REACTION study). Mod Rheumatol 2011;21:122-33.

4. Silpa-Archa S, Oray M, Preble JM, Foster CS. Outcome of tocilizumab treatment in refractory ocular inflammatory diseases. Acta Ophthalmol 2016;94:e400-6.

5. Tode J, Zeuner R, Saeger M, Roider J, Nolle B. Tocilizumab and steroid boli for treatment-resistant anterior necrotizing scleritis. Acta Ophthalmol 2015;93:e683-4.

6. Shimizu H, Nishioka H. Successful treatment with tocilizumab for refractory scleritis associated with relapsing polychondritis. Scand J Rheumatol 2017;25:1-2.

7. Laurent S, Le Parc JM, Clerici T, Breban M, Mahe E. Onset of psoriasis following treatment with tocilizumab. Br J Dermatol 2010;163:1364-5.

8. Palmou-Fontana N, Sánchez Gaviño JA, McGonagle D, García-Martinez E, Iñiguez de Onzoño Martin L.

Tocilizumab-induced psoriasiform rash in rheumatoid arthritis. Dermatology 2014;228:311-13.

9. Nutz A, Pernet C, Combe, B, Cohen J. Sarcoidosis induced by tocilizumab: a paradoxical event? J Rheumatol 2013;40:1773-4.

10. Wendling D, Dernis E, Prati C, Frisch E, Delbosc B. Onset of inflammatory eye disease under tocilizumab treatment for rheumatologic conditions: a paradoxical effect? J Rheumatol $2011 ; 38: 2284$

J Rheumatol 2017;44:11; doi:10.3899/jrheum.170234 